\title{
Degree of differentiation of cutaneous squamous cell carcinoma: a comparison between a Swedish cohort of organ transplant recipients and immunocompetent patients
}

\author{
Caroline Stenman ${ }^{1}$, Helena Gonzalez ${ }^{1}$, Martin Gillstedt ${ }^{1}$, Göran Dellgren ${ }^{2,3}$, Bengt Hasséus ${ }^{4}$, \\ Erik Holmberg ${ }^{5}$, Helena Rexius ${ }^{2}$, Jenny Öhman ${ }^{4}$, John Paoli ${ }^{1}$
}

\footnotetext{
1 Department of Dermatology and Venereology, Institute of Clinical Sciences, Sahlgrenska Academy, University of Gothenburg, Gothenburg, Sweden

2 Department of Cardiothoracic Surgery and Transplant Institute, Sahlgrenska University Hospital, Gothenburg, Sweden

3 Department of Molecular and Clinical Medicine, Institute of Medicine, Sahlgrenska Academy, University of Gothenburg, Gothenburg, Sweden

4 Department of Oral Medicine and Pathology, Institute of Odontology, Sahlgrenska Academy, University of Gothenburg, Gothenburg, Sweden

5 Department of Oncology, Institute of Clinical Sciences, Sahlgrenska Academy, University of Gothenburg, Gothenburg Sweden
}

Key words: cutaneous squamous cell carcinoma, organ transplant recipients, immunosuppression, histopathological differentiation, nonmelanoma skin cancer

Citation: Stenman C, Gonzalez H, Gillstedt M, Dellgren G, Hasséus B, Holmberg E, Rexius H, Öhman J, Paoli J. Degree of differentiation of cutaneous squamous cell carcinoma: a comparison between a Swedish cohort of organ transplant recipients and immunocompetent patients. Dermatol Pract Concept. 2018;8(4):330-336. DOI: https://doi.org/10.5826/dpc.0804a18

Received: February 25, 2018; Accepted: May 23, 2018; Published: October 31, 2018

Copyright: $\odot 2018$ Stenmen et al. This is an open-access article distributed under the terms of the Creative Commons Attribution License, which permits unrestricted use, distribution, and reproduction in any medium, provided the original author and source are credited.

Funding: The study was financed by grants from the federal government of Sweden under the ALF agreement.

Competing interests: The authors have no conflicts of interest to disclose.

All authors have contributed significantly to this publication.

Corresponding author: John Paoli, Department of Dermatology, Sahlgrenska University Hospital, Gröna stråket 16, 41345 Gothenburg, Sweden. Email: john.paoli@vgregion.se

ABSTRACT Background: Organ transplant recipients (OTRs) have a very high risk of developing cutaneous squamous cell carcinoma (cSCC). Immunosuppressed OTRs may have a higher proportion of poorly differentiated cSCC than non-OTRs.

Objectives: The aim of this study was to investigate the degree of differentiation of cSCCs in OTRs compared with immunocompetent individuals.

Patients/Methods: Data from the Swedish Cancer Registry were crosschecked with data from the Transplant registry of the Transplant Institute at Sahlgrenska University Hospital in Gothenburg, Sweden. All OTRs with a diagnosis of cSCC, basosquamous carcinoma, and/or cSCC in situ established at the Department of Dermatology, Sahlgrenska University Hospital, during 2002-2015 were included. The control group consisted of non-OTRs with the same diagnoses during the same time period. 
ABSTRACT Results: During 2002-2015, 82 OTRs diagnosed with 515 tumors and 883 non-OTRs with 1,247 tumors were included. OTRs developed 0.47 tumors/year vs 0.10 tumors/year for non-OTRs, but no significant differences were observed in the degree of tumor differentiation of invasive cSCCs between OTRs and non-OTRs $(\mathrm{P}=0.4)$. The distribution of poorly, moderately, and well-differentiated invasive cSCCs among OTRs and non-OTRs were $8.5 \%$ vs $12.5 \%, 22.1 \%$ vs $29.9 \%$, and $69.4 \%$ vs $57.6 \%$, respectively.

Conclusions: OTRs do not develop a higher proportion of poorly differentiated cSCCs than nonOTRs.

\section{Introduction}

Organ transplant recipients (OTRs) have a well-known increased risk of developing cancer due to immunosuppression [1]. Nonmelanoma skin cancer, particularly cutaneous squamous cell carcinoma (cSCC), is the most common malignancy seen in OTRs. Compared with the general population, the risk of developing cSCC is 60 to 100 times greater in OTRs [2-4]. Furthermore, cSCCs in OTRs have been shown to be more numerous and more aggressive compared with cSCCs in immunocompetent individuals [5-7]. In addition, the incidence of cSCC is proportional to the level of immunosuppression and is often associated with human papillomavirus (HPV) infection [8]. The pathogenesis of cSCC is multifactorial. For both OTRs and immunocompetent individuals, the most important risk factor for development of cSCC is UV radiation [9]. Fair skin is also seen as a risk factor [10]. There is a strong correlation between age and cSCC, with the highest incidence rates observed in men and women over 85 years of age [11]. Another risk factor for cSCC is the presence of oncogenic viruses in the skin [12]. In recent years, HPV has been associated with cSCC in OTRs. Among these patients, $80 \%$ of cSCCs are associated with HPV infection, in contrast to only $40 \%$ among immunocompetent individuals [13]. Greater age at transplantation as well as higher doses and longer duration of immunosuppressive regimens have also been identified as risk factors $[9,14]$.

Histopathologically, cSCC originates from epidermal keratinocytes and consists of nests, sheets, and strands of epithelial cells that arise from the epidermis and invade into the dermis for a variable distance. The cSCCs are commonly divided into 3 categories: well differentiated, moderately differentiated, and poorly differentiated depending on the resemblance of the tissue of origin. There are no clear objective parameters for grading the differentiation of cSCCs [15], but one classification is that $>75 \%$ of the cells are differentiated in well-differentiated tumors, $25 \%-75 \%$ in moderately differentiated tumors, and $<25 \%$ in poorly differentiated tumors [7]. Well-differentiated cSCCs tend to grow at a slower rate and metastasize to a lesser extent than tumors that are poorly differentiated [16].
One may hypothesize that cSCCs in OTRs tend to have a higher proportion of poorly differentiated tumors than cSCCs in immunocompetent patients, which could also contribute to an increased mortality. To investigate this, we aimed to study the degree of differentiation of cSCCs in immunosuppressed OTRs compared with immunocompetent individuals.

\section{Materials and Methods}

Gothenburg's Regional Ethical Review Board approved the study.

\section{Study Population}

Data pertaining to all diagnosed skin cancers were obtained from the Swedish Cancer Registry of the National Board of Health and Welfare, which contains data on all cancers diagnosed among the Swedish population since 1958 [17]. The transplant registry at the Transplant Institute at Sahlgrenska University Hospital (SUH) contains data regarding all patients who received transplants from January 1965 at SUH. This registry was used to acquire data on the OTRs who underwent organ transplantation between 1965 and 2010.

The International Statistical Classification of Diseases (ICD-10) codes C44.0S-C44.9S (cSCC), D04.0-D04.9 (cSCC in situ), and Z08.9B (follow-up visit for cSCC) were used to identify patients.

Patients from the transplant registry were crosschecked with patients from the Swedish Cancer Registry in order to find OTRs with an ICD code for cSCC in situ and/or cSCC. Tumors occurring prior to the first transplantation were excluded. Only patients diagnosed at the Department of Dermatology, SUH from 2002 (the year the electronic chart system was introduced) but prior to May 31, 2015, were included in this group, a total of 82 patients with 515 tumors. The control group consisted of non-OTRs diagnosed with cSCC and/or cSCC in situ at the Department of Dermatology, SUH from 2002 to May 31, 2015. A total of 883 patients with 1,247 tumors were included.

The histopathological report for each tumor was collected from the electronic chart system at SUH. The tumors were classified into 6 groups: cSCC in situ, well-differentiated 
TABLE 1. Demographic statistics and number of tumors per patient in the OTR and non-OTR groups

\begin{tabular}{|c|c|c|c|c|c|c|c|c|c|c|}
\hline & \multicolumn{5}{|c|}{ OTRs $(n=82)$} & \multicolumn{5}{|c|}{ Non-OTRs $(n=883)$} \\
\hline & \multirow{2}{*}{ Mean } & \multirow{2}{*}{ Median } & \multirow{2}{*}{ SD } & \multicolumn{2}{|c|}{$95 \% \mathrm{Cl}$} & \multirow{2}{*}{ Mean } & \multirow{2}{*}{ Median } & \multirow{2}{*}{ SD } & \multicolumn{2}{|c|}{$95 \% \mathrm{Cl}$} \\
\hline & & & & Lower & Upper & & & & Lower & Upper \\
\hline $\begin{array}{l}\text { Age (years) at First Dia } \\
(2002-2015)\end{array}$ & & & & & & & & & & \\
\hline Men & 61 & 62 & 10 & 57 & 64 & 78 & 80 & 10 & 77 & 79 \\
\hline Women & 57 & 59 & 13 & 53 & 61 & 80 & 82 & 12 & 79 & 81 \\
\hline All & 59 & 61 & 12 & 57 & 62 & 79 & 81 & 11 & 78 & 80 \\
\hline $\begin{array}{l}\text { No. of Tumors/Patient } \\
(2002-2015)\end{array}$ & 6.3 & 3.0 & 7.3 & 4.7 & 7.9 & 1.4 & 1.0 & 1.6 & 1.3 & 1.5 \\
\hline
\end{tabular}

cSCC, moderately differentiated cSCC, poorly differentiated cSCC, and "cSCC, other" (including cases of invasive or microinvasive cSCC without a specified degree of differentiation, cSCC and basosquamous carcinoma). In some cases, the same tumor had several histopathological reports, eg, the first report was from a punch biopsy and the second report from when the tumor was excised in toto. In such cases, the lowest differentiation level was chosen, and the same tumor is represented only once in the material.

\section{Statistical Analysis}

All data were analyzed using R version 3.0.3 (The R Foundation for Statistical Computing, Vienna, Austria) and Stata version 13.1 (STATA Corporation, College Station, TX). The Fisher exact test was used to compare proportions. The Mantel-Haenszel test was used to compare proportions across groups stratifying with respect to age group $(<55$ years, 55-64 years, and $\geq 65$ years). The Wilcoxon rank sum test was used for 2-sample tests. All tests are 2-sided. P values $<0.05$ and $95 \%$ confidence intervals (CI) were considered statistically significant.

Relative survival was computed using the Ederer II method [18]. Mortality data for the general population in Sweden were used to estimate expected survival rates for the study populations. The mortality data contained the probability of death for single-year age groups and gender in 1-year calendar periods. The strs macro, developed by Paul Dickman, Enzo Coviello, and Michael Hills, in the Stata statistical software, version 13.1, was used for the calculation of the relative survival. Survival time was calculated from date of diagnosis to date of death or to December 31, 2016.

\section{Results}

\section{Analysis of Study Population}

Patient characteristics are shown in Table 1 . A total of 82 OTRs were included, of which 36 (44\%) were women and $46(56 \%)$ were men. The mean and median ages at diagnosis were 59 years and 61 years, respectively (range: 31-82 years). The OTR cohort comprised 70 kidney, 6 liver, 5 heart, and 1 lung recipient. The mean and median time since transplantation was 15 and 14 years, respectively (range: 0.435 years). The average follow-up time was 13.5 years.

In the non-OTR group, 883 patients were included, comprising 405 (46\%) women and 478 (54\%) men. The mean and median ages at diagnosis were 79 and 81 years, respectively (range: 32-101 years). The average follow-up time was 13.5 years.

\section{cSCC and cSCC In Situ}

The tumor characteristics for both groups are presented in Table 2. The degrees of differentiation of the invasive cSCCs in OTRs compared with non-OTRs are shown in Figure 1.

In the OTR group, a total of 515 tumors were found with an average of 6.3 tumors/patient. The average follow-up time was 13.5 years, which corresponds to 0.47 tumors/year. Of the 515 tumors, 198 were cSCC in situ and 258 were cSCC with a known degree of differentiation. In these cases, 22 $(8.5 \%)$ were poorly differentiated, $57(22.1 \%)$ were moderately differentiated, and 179 (69.4\%) were well differentiated. The number of tumors in the non-OTR group was 1,247, an average of 1.4 tumors/patient and 0.10 tumors/year. Among these tumors, 86 were cSCC in situ and 1,019 were cSCC with a known degree of differentiation: $127(12.5 \%)$ were poorly differentiated, $305(29.9 \%)$ were moderately differentiated, and $587(57.6 \%)$ were well differentiated. When comparing the distribution of the level of differentiation of the cSCCs with available data while also stratifying with respect to age group between the OTRs and non-OTRs, no significant difference was seen (P value 0.4, Mantel-Haenszel test).

As an incidental finding, an association was seen between the distribution of the level of differentiation of cSCCs and age groups below and above the median age at diagnosis in both groups. OTRs who were above the median age of 62 years had significantly fewer well-differentiated cSCCs (61\%, CI 52-70) than younger OTRs (76\%, CI 69-83) 
TABLE 2. Descriptive statistics regarding the distribution of the different types of cSCC and the anatomic location of the tumors in the OTR and non-OTR groups

\begin{tabular}{|c|c|c|c|c|}
\hline & \multicolumn{2}{|c|}{ Tumors in OTRs $(n=515)$} & \multicolumn{2}{|c|}{ Tumors in non-OTRs $(n=1,247)$} \\
\hline & $\mathbf{n}$ & $\%$ & $\mathbf{n}$ & $\%$ \\
\hline \multicolumn{5}{|l|}{ Tumor Type } \\
\hline cSCC in situ & 198 & $38 \%$ & 86 & $7 \%$ \\
\hline Well-differentiated cSCC & 179 & $35 \%$ & 587 & $47 \%$ \\
\hline Moderately differentiated cSCC & 57 & $11 \%$ & 305 & $24 \%$ \\
\hline Poorly differentiated cSCC & 22 & $4 \%$ & 127 & $10 \%$ \\
\hline cSCC, other* & 59 & $11 \%$ & 142 & $11 \%$ \\
\hline \multicolumn{5}{|l|}{ Anatomic Location } \\
\hline Head or neck & 175 & $34 \%$ & 738 & $59 \%$ \\
\hline Upper extremity & 140 & $27 \%$ & 121 & $10 \%$ \\
\hline Lower extremity & 86 & $17 \%$ & 187 & $15 \%$ \\
\hline Trunk & 114 & $22 \%$ & 199 & $16 \%$ \\
\hline Unknown & 0 & $0 \%$ & 2 & $0 \%$ \\
\hline \multicolumn{5}{|c|}{ Anatomic Location (Only Invasive cSCC) } \\
\hline Head or neck & 116 & $38 \%$ & 689 & $60 \%$ \\
\hline Upper extremity & 90 & $29 \%$ & 107 & $9 \%$ \\
\hline Lower extremity & 47 & $15 \%$ & 167 & $15 \%$ \\
\hline Trunk & 56 & $18 \%$ & 186 & $16 \%$ \\
\hline Unknown & 0 & $0 \%$ & 2 & $0 \%$ \\
\hline \multicolumn{5}{|l|}{ Anatomic Location (Only cSCC in situ) } \\
\hline Head or neck & 56 & $28 \%$ & 42 & $49 \%$ \\
\hline Upper extremity & 49 & $25 \%$ & 14 & $16 \%$ \\
\hline Lower extremity & 38 & $19 \%$ & 18 & $21 \%$ \\
\hline Trunk & 55 & $28 \%$ & 12 & $14 \%$ \\
\hline Unknown & 0 & $0 \%$ & 0 & $0 \%$ \\
\hline
\end{tabular}

* Includes 8 basosquamous carcinomas in OTRs and 10 basosquamous carcinomas in non-OTRs.

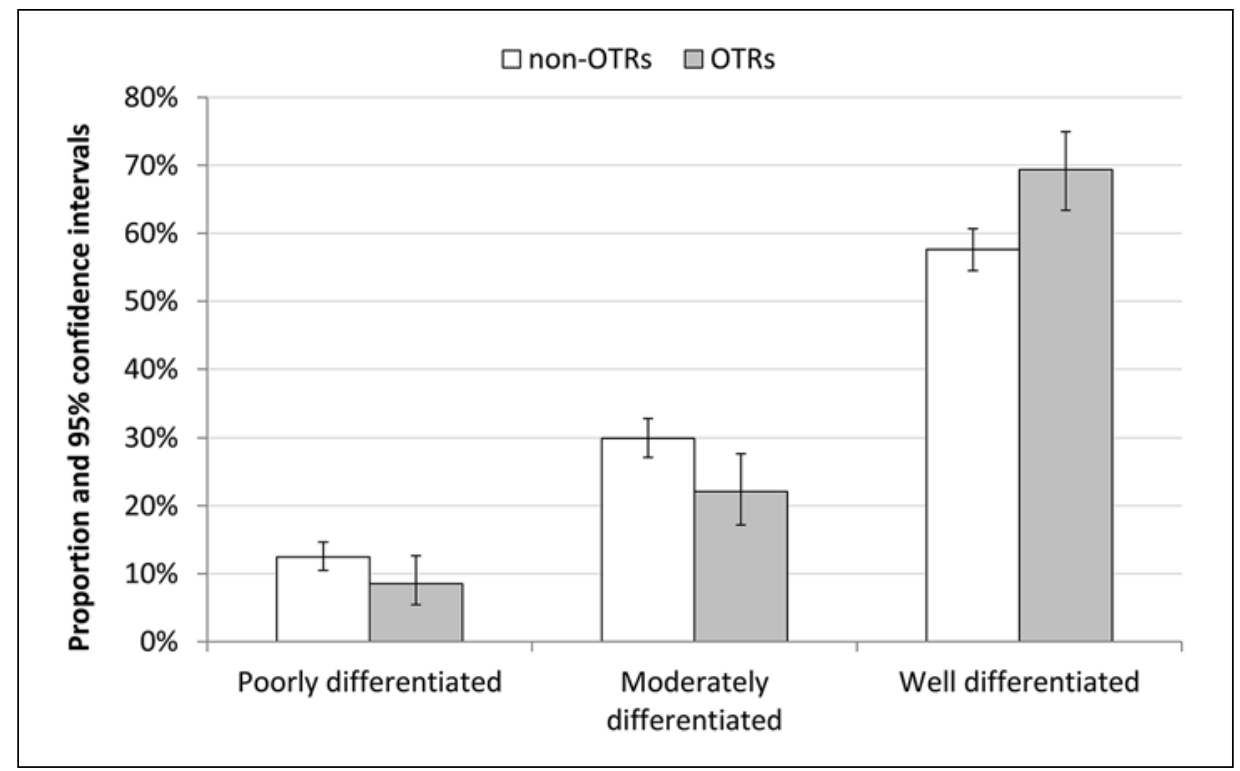

Figure 1. Distribution of the degrees of differentiation for the OTR and non-OTR groups. The vertical lines within the bars denote 95\% confidence intervals. [Copyright: @2018 Stenmen et al.] 


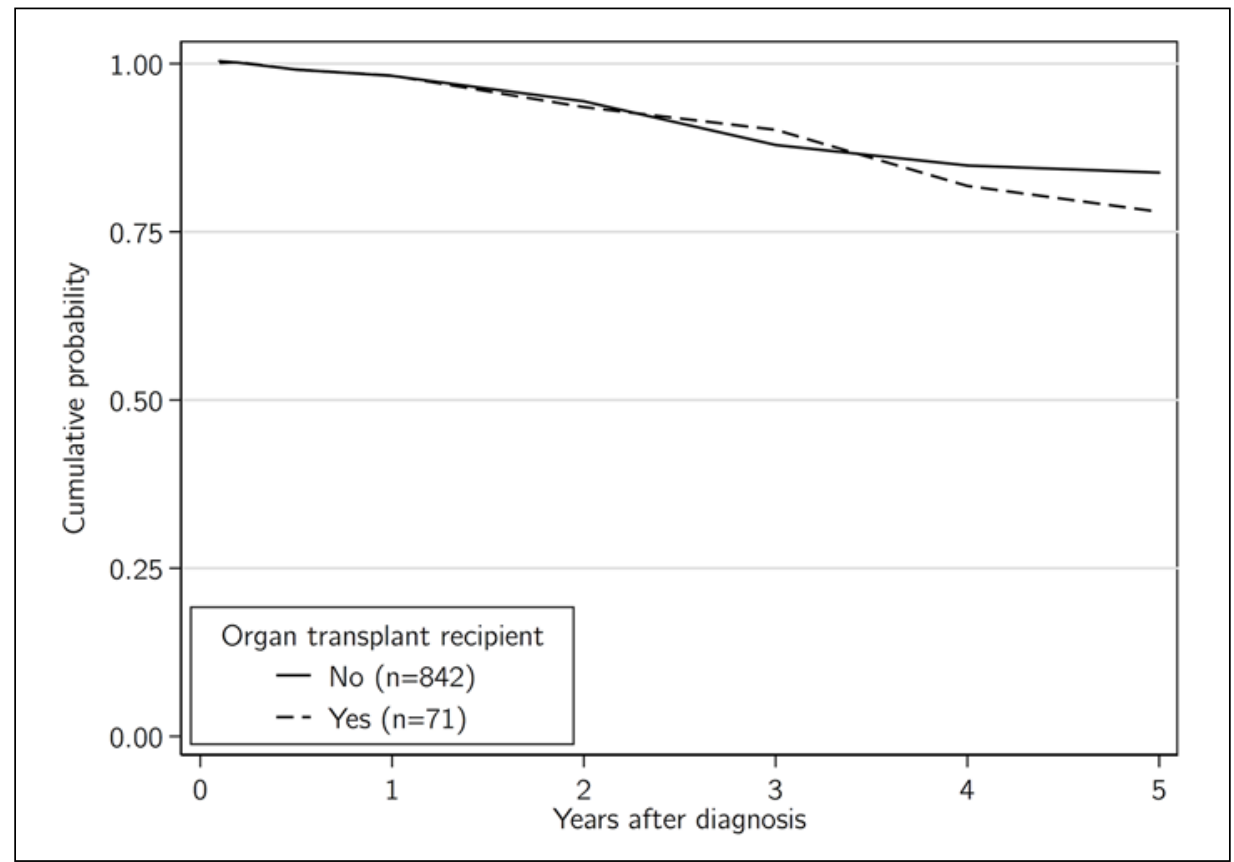

Figure 2. Relative survival comparing OTRs and non-OTRs who had $\geq 1$ invasive cSCC. [Copyright: C2018 Stenmen et al.]

(P value 0.03, Fisher exact test). The same phenomenon was seen among the non-OTRs when looking at patients above the median age of 81 years with $51 \%$ (CI 46-55) of cSCCs being well differentiated compared with $64 \%$ (CI 60-68) in non-OTRs below the median age ( $\mathrm{P}$ value 0.0005 , Fisher exact test).

Furthermore, OTRs were diagnosed with significantly more cSCC in situ ( $\mathrm{n}=198,0.18$ per person and year) than the non-OTRs ( $\mathrm{n}=86,0.01$ per person and year) $(\mathrm{P}<0.0001)$ despite the 20 -year difference in mean age between the groups (59 years for OTRs and 79 years for non-OTRs).

\section{Tumor Site}

Another incidental finding was the distribution of the anatomic location of the tumors in the 2 groups. The tumor site differed significantly between the OTR and the non-OTR groups when stratifying for age group $(\mathrm{P}<0.0001$, MantelHaenszel test). OTRs had a significantly larger proportion of tumors on the upper extremities $(27 \%$ vs $10 \%, \mathrm{P}<0.0001)$ and the trunk $(22 \%$ vs $16 \%, P=0.003)$. In contrast, the non-OTRs had a significantly higher proportion of tumors in the head and neck area $(59 \%$ vs $34 \%, \mathrm{P}<0.0001)$. When comparing the anatomic location of the tumors between the OTR and non-OTR groups for invasive cSCCs and cSCC in situ lesions separately, the significant difference remained ( $\mathrm{P}$ $<0.0001$ for invasive cSCCs and $\mathrm{P}=0.003$ for the in situ lesions). When comparing the proportion of lesions on the head and neck between OTRs and non-OTRs, the significant difference also remained for invasive cSCCs (OTRs 38\% and
non-OTRs $60 \%, \mathrm{P}<0.0001$ ) and cSCC in situ (OTRs $28 \%$ and non-OTRs $49 \%, P=0.001)$. There was no significant difference in the degree of differentiation for cSCCs in the head and neck area when comparing OTRs and non-OTRs. However, cSCCs in the head and neck area showed significantly poorer tumor differentiation compared with cSCCs on other body sites, when stratifying with respect to age groups $(\mathrm{P}<0.00001)$.

\section{Survival Analysis}

The relative survival showed no difference between the 2 groups (Figure 2). For non-OTRs, the relative survival was 0.84 (CI 0.78-0.89) and for OTRs it was 0.78 (CI 0.65-0.88).

\section{Discussion}

The correlation between immunosuppression and skin cancer has been extensively studied [1,3,4], but far less is known about the difference in degree of differentiation of cSCCs in OTRs as compared with cSCCs in immunocompetent patients. To our knowledge, only Harwood et al and, more recently, Cheng et al have previously compared the degree of differentiation of cSCCs among OTRs and non-OTRs, finding no significant differences in smaller series $[19,20]$.

There are several studies that show a more lethal and more aggressive behavior of cSCCs in OTRs [7,21]. However, $69.4 \%$ of cSCCs in OTRs in this study were well differentiated, which is consistent with the results from Lindelöf et al showing an almost identical proportion of well-differentiated 
cSCCs $(68.3 \%)$ [22]. In comparison with the non-OTRs, there was no significant difference in the degree of tumor differentiation. In fact, there was a slightly higher proportion of poorly differentiated cSCCs among non-OTRs (not significant).

As an incidental finding, we discovered that there was a higher frequency of poorly differentiated cSCCs in the patients above the median age, independently of whether the patient was an OTR or not. The fact that OTRs acquire their tumors at a much younger age (20 years earlier than the nonOTRs) may explain the finding that OTRs have a relatively small proportion of poorly and moderately differentiated tumors. Our findings corroborate a study by Harwood et al in which OTRs were 15 years younger than the non-OTRs [19].

OTRs developed on average 6.3 tumors per patient during an average follow-up time of 13.5 years, which gave a number of tumors per patient-year of 0.47 . This high number of cSCCs per patient-year is most probably due to the immunosuppressive medication [1,2,23], but early diagnoses thanks to closer follow-up visits may also play a role among OTRs [23]. Notably, cSCC in situ was significantly more common in OTRs. This has also been seen in other studies [2,24].

In a Swedish study from 2000, 172 OTRs had a total of 325 nonmelanoma skin cancers (NMSCs), not including basal cell carcinomas. The average follow-up time was 9.2 years. For these patients with NMSCs, the number of NMSCs per person-year was 0.20 [1].

In a study from the United Kingdom, 257 OTRs had 622 NMSCs including cSCC in situ and basal cell carcinomas. The follow-up time was 8 years and the number of NMSCs per person-year was 0.30 [2]. The higher number of tumors per person-year in our study may be explained by the fact that the study period was more recent and the incidence of cSCC in Sweden has increased during the past decade [25]. The patients in our study had also been immunosuppressed for a longer period of time (13.5 years vs 9.2 years). Furthermore, the knowledge about the risk of cSCC in OTRs has increased and nowadays there is a much more organized system for follow-up visits which might have resulted in more diagnosed cases per patient.

Surprisingly, non-OTRs had a significantly higher proportion of tumors in the head and neck area compared with OTRs and the significance remained when comparing the anatomic location of the tumors between the groups for cSCCs and cSCC in situ lesions separately. All patients who undergo transplantation at SUH receive information about the risks of sun exposure. A hypothesis may be that OTRs therefore use sunscreen on the chronically exposed facial skin more frequently than non-OTRs. Another explanation could be that UV radiation is not the only risk factor for cSCCs in OTRs. In OTRs, $80 \%$ of cSCCs are associated with HPV infection, in contrast to only $40 \%$ among immunocompetent individuals [13].

With regard to the relative survival, no significant difference was seen between OTRs and non-OTRs, but the comparison is difficult to make due to the large difference in mean age between the groups.

A strength of this study is that the conditions for population studies in Sweden are ideal, thanks to a history of extensive record-keeping and the fact that each citizen is assigned a unique social security number at birth, allowing all citizens to be traced through different records over time across the whole country without missing data, except if patients emigrate. There are, however, some limitations as well. Among both OTRs and non-OTRs, $11 \%$ of the invasive cSCCs had an unknown degree of differentiation. We were also confined to the use of the electronic chart system, which was implemented at SUH in 2002. Since there is no national central medical record, we were not able to follow up with patients who may have been diagnosed with cSCC at other clinics. Finally, other factors that may also contribute to the aggressiveness of cSCCs (eg, tumor depth) were not analyzed.

\section{Conclusions}

There was no significant difference between the groups with regard to the proportion of poorly differentiated cSCCs. OTRs develop a significantly higher number of cSCCs and SCC in situ than non-OTRs. These tumors are more often located on the trunk and upper extremities in OTRs, whereas non-OTRs develop more tumors on the chronically UVexposed skin of the head and neck area. Although cSCCs in OTRs have traditionally been considered more aggressive, the larger number of cSCCs in OTRs might be what increases the risk for recurrences and metastases and not the intrinsic malignant potential of each individual cSCC per se.

\section{Acknowledgments}

We thank Dr. Britta Krynitz for her valuable input and discussions surrounding the histopathology of cutaneous squamous cell carcinoma.

\section{References}

1. Lindelöf B, Sigurgeirsson B, Gabel H, Stern RS. Incidence of skin cancer in 5356 patients following organ transplantation. $\mathrm{Br} \mathrm{J}$ Dermatol. 2000;143(3):513-519.

2. Moloney FJ, Comber H, O'Lorcain P, O'Kelly P, Conlon PJ, Murphy GM. A population-based study of skin cancer incidence and prevalence in renal transplant recipients. $\mathrm{Br} J$ Dermatol. 2006;154(3):498-504. 
3. Krynitz B, Edgren G, Lindelöf B, et al. Risk of skin cancer and other malignancies in kidney, liver, heart and lung transplant recipients 1970 to 2008: a Swedish population-based study. Int J Cancer. 2013;132(6):1429-1438.

4. Euvrard S, Kanitakis J, Claudy A. Skin cancers after organ transplantation. N Engl J Med. 2003;348(17):1681-1691.

5. Boyle J, MacKie RM, Briggs JD, Junor BJ, Aitchison TC. Cancer, warts, and sunshine in renal transplant patients: a case-control study. Lancet. 1984;1(8379):702-705.

6. Gupta AK, Cardella CJ, Haberman HF. Cutaneous malignant neoplasms in patients with renal transplants. Arch Dermatol. 1986;122(11):1288-1293.

7. Lindelöf B, Jarnvik J, Ternesten-Bratel A, Granath F, Hedblad MA. Mortality and clinicopathological features of cutaneous squamous cell carcinoma in organ transplant recipients: a study of the Swedish cohort. Acta Derm Venereol. 2006;86(3):219-222.

8. Buell JF, Gross TG, Woodle ES. Malignancy after transplantation. Transplantation. 2005;80(2 suppl):S254-S264.

9. Vajdic CM, van Leeuwen MT. Cancer incidence and risk factors after solid organ transplantation. Int J Cancer. 2009;125(8):17471754.

10. English DR, Armstrong BK, Kricker A, Winter MG, Heenan PJ, Randell PL. Demographic characteristics, pigmentary and cutaneous risk factors for squamous cell carcinoma of the skin: a case-control study. Int J Cancer. 1998;76(5):628-634.

11. Hussain SK, Sundquist J, Hemminki K. Incidence trends of squamous cell and rare skin cancers in the Swedish National Cancer Registry point to calendar year and age-dependent increases. $J$ Invest Dermatol. 2010;130(5):1323-1328.

12. Penn I. Cancers in renal transplant recipients. Adv Ren Replace Ther. 2000;7(2):147-156.

13. Accardi R, Gheit T. Cutaneous HPV and skin cancer. Presse Med. 2014;43(12 pt 2):e435-e443.

14. Webb MC, Compton F, Andrews PA, Koffman CG. Skin tumors posttransplantation: a retrospective analysis of 28 years' experience at a single centre. Transplant Proc. 1997;29(1-2):828-830.
15. Weedon D, Morgan MB, Gross C, Nagoro E, Yu LL. Squamous cell carcinoma. In: LeBoit PE, ed. Pathology and Genetics of Tumors of the Skin. Lyon: IARC Press; 2006:20-25.

16. Kumar V, Abbas AK, Fausto N, Robbins SL, Cotran RS. Pathologic Basis of Disease Vol 7. Robbins SL, Cotran RS, eds. Philadelphia: Elsevier Saunders; 2005.

17. Swedish National Board of Health and Welfare. Cancer incidence in Sweden 2011. 2012; Available at: https://www.socialstyrelsen. se/Lists/Artikelkatalog/Attachments/18919/2012-12-19.pdf. Accessed February 25, 2018.

18. Ederer F, Axtell LM, Cutler SJ. The relative survival rate: a statistical methodology. Natl Cancer Inst Monogr. 1961 Sep;6:101-21.

19. Harwood CA, Proby CM, McGregor JM, Sheaff MT, Leigh IM, Cerio R. Clinicopathologic features of skin cancer in organ transplant recipients: a retrospective case-control series. J Am Acad Dermatol. 2006;54(2):290-300.

20. Cheng JY, Li FY, Ko CJ, Colegio OR. Cutaneous squamous cell carcinomas in solid organ transplant recipients compared with immunocompetent patients. JAMA Dermatol. 2018;154(1):60-66.

21. Berg D, Otley CC. Skin cancer in organ transplant recipients: epidemiology, pathogenesis, and management. J Am Acad Dermatol. 2002;47(1):1-17; quiz 18-20.

22. Lindelöf B, Dal H, Wolk K, Malmborg N. Cutaneous squamous cell carcinoma in organ transplant recipients: a study of the Swedish cohort with regard to tumor site. Arch Dermatol. 2005;141(4):447-451.

23. Ramsay HM, Fryer AA, Hawley CM, Smith AG, Nicol DL, Harden PN. Factors associated with nonmelanoma skin cancer following renal transplantation in Queensland, Australia. J Am Acad Dermatol. 2003;49(3):397-406.

24. Ramsay HM, Fryer AA, Reece S, Smith AG, Harden PN. Clinical risk factors associated with nonmelanoma skin cancer in renal transplant recipients. Am J Kidney Dis. 2000;36(1):167-176.

25. Swedish National Board of Health and Welfare. Cancer incidence in Sweden 2014. 2015; Available at: http://www.socialstyrelsen. se/Lists/Artikelkatalog/Attachments/20008/2015-12-26.pdf. Accessed February 25, 2018. 